\title{
Interspecific delimitation and relationships among four Ostrya species based on plastomes
}

\author{
Yanyou Jiang, Yongzhi Yang, Zhiqiang Lu, Dongshi Wan and Guangpeng Ren * (D)
}

\begin{abstract}
Background: The genus Ostrya (Betulaceae) contains eight species and four of them are distributed in China. However, studies based on limited informative sites of several chloroplast markers failed to resolve interspecific delimitation and relationships among the four Chinese species. In this study, we aimed to use the whole chloroplast genomes to address these two issues.

Results: We assembled and annotated 33 complete chloroplast genomes (plastomes) of the four Chinese species, representing 17 populations across most of their geographical distributions. Each species contained samples of several individuals that cover most of geographic distributions of the species. All plastomes are highly conserved in genome structure and gene order, with a total length of 158-159 kb and 122 genes. Phylogenetic analyses of whole plastomes, non-coding regions and protein-coding genes produced almost the same topological relationships. In contrast to the well-delimitated species boundary inferred from the nuclear ITS sequence variations, three of the four species are non-monophyletic in the plastome trees, which is consistent with previous studies based on a few chloroplast markers.

Conclusions: The high incongruence between the ITS and plastome trees may suggest the widespread occurrences of hybrid introgression and incomplete lineage sorting during the divergence of these species. In addition, the plastomes with more informative sites compared with a few chloroplast markers still failed to resolve the phylogenetic relationships of the four species, and further studies involving population genomic data may be needed to better understand their evolutionary histories.
\end{abstract}

Keywords: Chloroplast genome, Interspecific relationship, Ostrya, Phylogeny, Species delimitation

\section{Background}

Ostrya is a small genus belongs to the birch family Betulaceae and merely consists of eight species native in southern Europe, southwest and eastern Asia, and North and Central America [1-3]. These small deciduous trees, commonly called as hophornbeam or ironwood, are well known for their hard and heavy woods. Ostrya woods have been used for various purposes such as furniture, axles, fuel wood and charcoal [2, 4]. It had been long thought that there are five species distributed in China (http://www.efloras.org) until Lu et al. (2016) [5] found that Ostrya yunnanensis was nested within O. multinervis

\footnotetext{
* Correspondence: rengp@|zu.edu.cn

State Key Laboratory of Grassland Agro-Ecosystem, School of Life Sciences, Lanzhou University, Lanzhou, Gansu, People's Republic of China
}

in both phylogenetic trees and morphological clustering. The two species were therefore recognized as the same species and renamed as O. chinensis [6]. Among the four species, $O$. japonica is mainly distributed in China, Japan and Korea, while the remaining three species, O. rehderi$a n a, O$. chinensis and $O$. trichocarpa, are endemic to China. The amount of these wild plants has been decreased rapidly due to overexploitation, habitat destruction and ecosystem deterioration. The famous endangered species, O. rehderiana, has been reported that only five mature trees survive and are conserved in the Tianmu Mountain, Zhejiang [7]. Although Ostrya species own excellent wood and are regarded as ecologically and economically valuable foundation for future forests, the interspecific delimitation and relationships remain unresolved. Previous study failed to discern the four Ostrya 
species based on four chloroplast (cp) fragments (rps16, $\operatorname{trn} G^{(\mathrm{UCC})}$ intron, $\operatorname{trn} H-p s b A$, and $\operatorname{trn} L-\operatorname{trn} F$ ) while the ITS sequence variations did [5]. It has been suggested that the traditionally accepted cpDNA barcodes own limited resolution in delimitating closely related species due to the insufficient informative variations [8-10]. By contrast, the complete chloroplast genomes (plastomes) were proven to provide more valuable information in revealing phylogeny of plants [11-16]. And with the development of Next-generation sequencing technology, it has recently become cost-effective and it is easier to sequence the complete plastomes in plants than before $[12,17,18]$. The plastomes with more informative sites may increase resolution of the previously unresolved phylogenetic relationships based on a few cpDNA markers [15, 19]. Therefore, in this study, we re-investigate phylogenetic relationships of the four Chinese Ostrya species based on plastomes. We selected multiple individuals per species to cover the most of geographic distributions of the species to reduce the sampling bias. We also extracted three datasets, i.e., the whole plastomes, non-coding regions and protein-coding genes, for phylogenetic analyses and compared these plastome trees with the ITS tree. Specifically, the aims of our study are to: 1) investigate whether the three plastome datasets result in consistent phylogenies and whether they can discern the four species and 2) test if phylogeny based on plastome datasets congruent with the ITS tree.

\section{Methods}

\section{Plant materials, DNA extraction and sequencing}

We chose in total 33 individuals from 17 populations representing all the four Ostrya species that occur in China. For each species, we selected samples that cover most of its geographic distribution (Additional file 1: Table S1). The fresh leaf materials were collected in the field and preserved with silica gel immediately. Total genomic DNA was extracted from $20 \mathrm{mg}$ silica gel-dried leaves on the basis of the modified CTAB procedure [20]. According to the basic protocol, we prepared the end-repaired, phosphorylated and A-tailed DNA fragments and ligated with index adapters. The library construction and whole-genome sequencing were accomplished at Beijing Genomics Institute (Shenzhen, Guangdong, China). The libraries were sequenced on the Illumina Hiseq 2000 platform.

\section{Genome assembly and annotation}

Raw reads of all samples were trimmed with a filter standard $(\mathrm{Q}<=5$ or $\mathrm{N}$ base content $>10 \%)$. Afterwards, we used Trimmomatic [21] to further filter the data. We downloaded about 3000 published chloroplast genome sequences from NCBI and built an index of them. All the high quality reads were then mapped to the index using Bowtie2 [22] and sorted by Samtools v.1.2 [23].
We used the software bam2fastq (https://gsl.hudsonalpha.org/information/software/bam2fastq) to generate the fastq format files from the bam file. Then, we assembled the plastomes using Velvet v1.2.10 [24] and filled up the gaps by GapCloser v1.12 (https://sourceforge.net/ projects/soapdenovo2/files/GapCloser/). Moreover, we used Plann v.1.1.2 [25] to annotate the plastomes using $O$. rehderiana as a reference. Then we corrected the start codons, stop codons and intron/exon boundaries manually using Sequin v.15.10 (http://www.ncbi.nlm.nih.gov/Sequin/) as well as a visual software Geneious v.R.8.1.4 [26]. Ultimately, the circular images were performed through OGDRAWv.1.1 (http://ogdraw.mpimp-golm.mpg.de/) [27].

\section{Genome comparison and genome repeat}

In order to show interspecific variations, the alignments of all plastomes were plotted using the mVISTA program with LAGAN mode [28]. All validated plastome sequences were submitted to GenBank (Additional file 2: Table S2). We determined three kinds of repeats among plastomes of the four Ostrya species: dispersed, palindromic and tandem repeat. The former two types were visualized and located by an online program REPuter [29] with a minimal length of $30 \mathrm{bp}$ and $90 \%$ sequence identities (Hamming distance equal to 3) between the two repeats [30]. Tandem Repeats Finder [31] was used to detect tandem repeats with the following parameters: Match 2, Mismatch 7, Delta 7, PM 80, PI 10, Minscore 50 , MaxPeriod 500, with similarity $100 \%$. We further verified all the repeats and removed the redundant segment manually.

\section{Phylogenetic analysis}

According to Grimm \& Renner (2013) [32], the most closely related genus Carpinus could not be separated well from Ostrya and there were no published Ostryopsis plastome sequences available in GenBank. We therefore downloaded a plastome of Corylus chinensis (GenBank accession number: NC_032351.1) from GenBank as outgroup to reconstruct the phylogenetic tree of the four Ostrya species. In order to test whether different plastome regions resulted in consistent phylogenies during the phylogenetic reconstructions, we used three plastome datasets: (1) the whole plastomes, (2) the non-coding regions and (3) the protein-coding genes (PCGs). In addition, we downloaded from GenBank the same ITS dataset as in Lu et al. (2016) [5]. For whole plastomes, non-coding sequences and ITS datasets, we used their nucleotide sequences directly and aligned each dataset using MAFFT v.7 [33] and trimal [34]. The later software was implemented for alignment trimming. Afterwards, we used Perl scripts to delete the gaps of aligned sequences. For the PCGs dataset, we first 
extracted 76 PCGs shared by all 33 Ostrya plastomes and Corylus chinensis and translated their nucleotide sequences into amino acid (aa) sequences for phylogenetic analysis in order to reduce the tree artifacts due to high DNA divergence. Multiple alignment of aa sequence of each gene was achieved by T-coffee [35] followed by trimal [34] to do automated alignment trimming. After that, FASconCAT-G [36] was used to produce a concatenated alignment.

Phylogenetic analyses were performed by both maximum likelihood (ML) and Bayesian inference (BI) methods. For whole plastomes, non-coding regions and ITS datasets, GTR $+\mathrm{I}+\mathrm{G}$ as the best-fit model selected by JModeltest v.2.1.1 [37] was used for both ML and BI analyses, which were conducted by RAxML v8.1.24 [38] and MrBayes 3.2.2 [39] respectively. For aa sequences of PCGs, Prottest v.3.4.2 [40] was applied to select best-fit aa substitution model for each gene. The selected best-fit models were listed in Additional file 3: Table S3. Then we performed ML analysis using RAxML v8.1.24 with specific aa substitution matrix for each partition by using the selected best-fit aa substitution model. As for BI analysis using MrBayes, preliminary searches (mcmc nchains $=1$; ngen $=10,000,000$ ) were made under mixed aamodel (preset aamodelpr $=$ mixed) to identify the best-fit aa substitution model. The result showed that Cprev was the best-fit aa substitution model. Then a final BI analysis using Cprev as the fixed model for all partitions (prset aamodel $=$ fixed $(\mathrm{Cprev})$ ) and unlinked model parameters for each partition were conducted in MrBayes.

The node supports were determined with 1000 bootstrap replicates in ML analyses. For BI analysis, two independent parallel runs and four chains (one cold and three hot) were running for 10,000,000 generations with trees sampled every 500 generations. Then we determined convergence by examining trace plots of the log likelihood values for each parameter in Tracer [41]. In addition, we calculated the distances among plastomes of the four Ostrya species using the web tool GGDC [42] (http://ggdc.dsmz.de/distcalc2.php) to further compare the inter- and intra-specific variation. As individuals from the same population were grouped together in the phylogenetic trees (see Results), only one individual from each population of the four species was selected for this analysis.

\section{Results}

\section{Plastome features}

Characteristics of Ostrya plastomes were conservative (Figs. 1 and 2) with the length ranged from 158,870159,301 bp. The chloroplast genome structures of all the four Ostrya species were consistent with mostly known angiosperms with a typical quadripartite structure consisting of a pair of inverted repeats regions (IRa and IRb:
26,059-26,069 bp) divided by a large single-copy region (LSC: 88,007-88,229 bp) and a small single-copy region (SSC: 18,721-18,975 bp) (Fig. 1 and Table 1). The overall GC content was absolutely identical (36.5\%; Table 1) across all plastomes. All plastomes possessed 122 unique genes, including 85 protein-coding genes (79 PCG species), 29 tRNA genes (24 tRNA species), and 8 ribosomal RNA genes (4 rRNA species, Table 1). Most genes appeared in a single copy, while 15 were replicated once on the IR regions, including 4 rRNA $(4.5 \mathrm{~S}, 5 \mathrm{~S}, 16 \mathrm{~S}$, and $23 \mathrm{~S}$ rRNA), 5 tRNA (trnI-CAT, trnL-CAA, trnV-GAC, trnR-ACG and trnN-GTT), and 6 PCG species (rpl2, rpl23, $y c f 2, n d h B, r p s 7$ and $y c f 1$; Additional file 4: Table S4). The rps12 gene was a unique trans-spliced gene with three exons. Expansion of IR regions into rps19 at the IRb/LSC boundary region occurred in all Ostrya taxa and the pseudogene $y c f 1$ was located at the junction of $\mathrm{SSC} / \mathrm{IRa}$, which gave rise to the incomplete duplication of protein-coding gene within IRs. In addition, among annotated genes, nine genes (rps16, atpF, rpoC1, petB, petD, rpl16, rpl2, $n d h B$ and $n d h A$ ) contained a single intron, and three genes (rps12, clpP and $y c f 3$ ) had two introns. Intergenic spacers showed moderate genetic divergences. The intergenic spacers of trnQ-psbK, trnS-trnR and $p s b Z$-trn $G$ were identified as the most divergence hotspots (Fig. 2).

Repeat sequences are considered to play a significant role in phylogenetic analysis and also make positive efficiency for genome rearrangement analysis [43, 44]. In this study, a total of 249 repeats were detected in Ostrya plastomes (Fig. 3 and Additional file 5: Table S5). All repeats were similar among the four species and their overall distributions were conserved (Fig. 3a). The length of repeated sequences was mainly concentrated from 30 bp to $44 \mathrm{bp}$ (Fig. 3b). The number of the three kinds of repeats was also similar, with palindromic repeat exhibiting maximum amounts (36.4\%) followed by dispersed (32.4\%) and tandem (31.2\%) types (Fig. 3c). Most repeats were detected in intergenic regions (57.1\%), while only a minority of repeats were distributed in intron regions (5.2\%). The remaining repeats $(37.7 \%)$ were found in coding regions (e.g., $y c f 1, y c f 2$, atpA, rpl2, rpl23, rps19, rps7, rps12 and $n d h B$; Fig. 3d).

\section{Phylogenetic analysis}

Three plastome datasets (i.e. whole plastomes, PCGs and non-coding regions) and one nuclear ITS sequences were used in this study for phylogenetic analyses. The whole plastome dataset comprised 157,116 bp, 2209 of which were variable and 456 were parsimony-informative. The non-coding dataset comprised 64,479 bp, 1332 of which were variable and 244 were parsimony-informative. 76 PCGs shared by all Ostrya samples and outgroup were translated into an amino acid dataset, which contained 


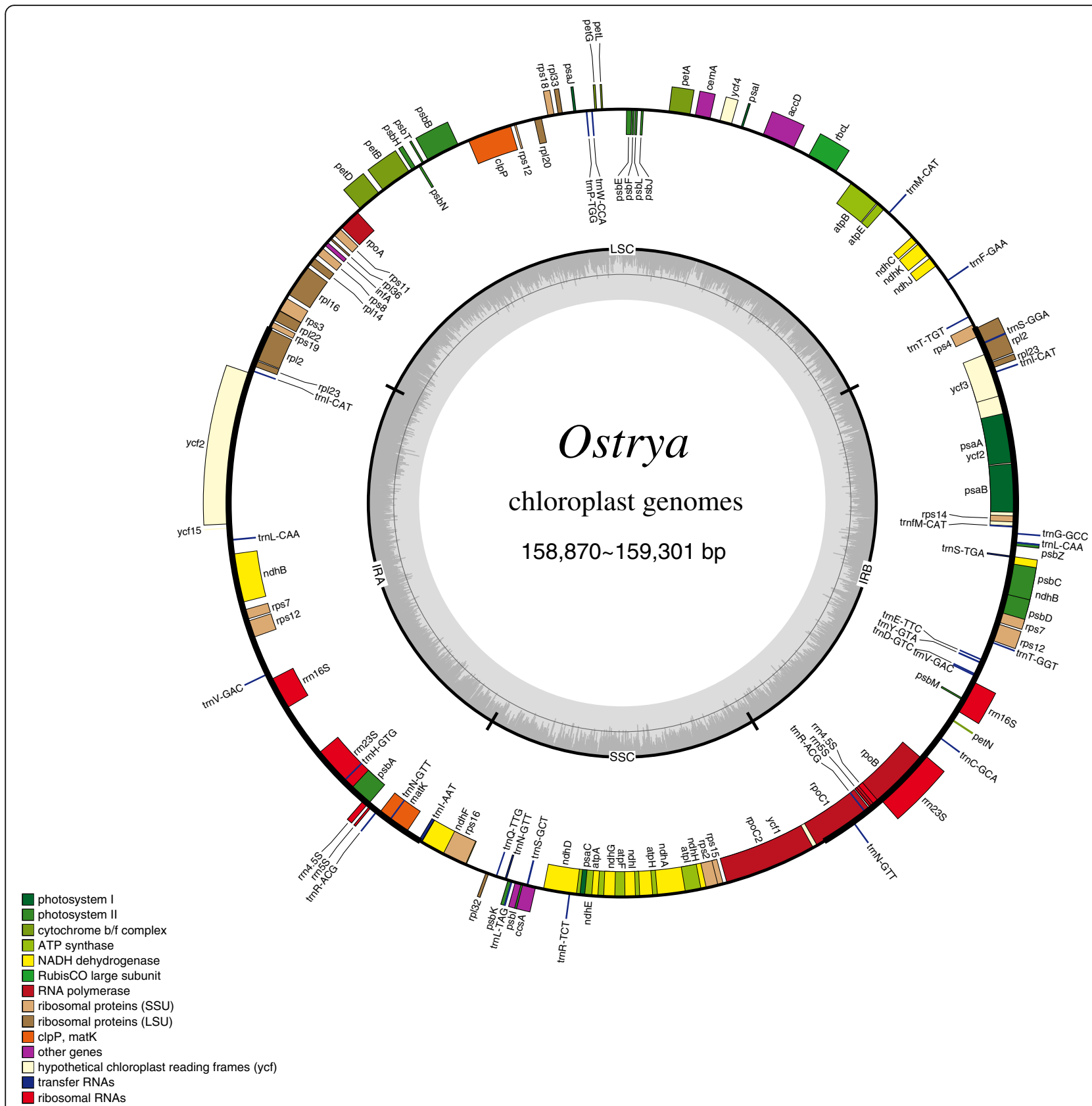

Fig. 1 Gene map of the Ostrya chloroplast genomes. Because all 33 platomes have identical gene content and gene number, here we only show one plastome map as an example. Genes inside and outside of the circle are transcribed counterclockwise and clockwise directions, respectively. Genes belonging to different functional groups are shown in different colors. The dark gray inner circle corresponds to the GC content and the light-gray circle corresponds to the AT content

21,720 aa, 228 of which were variable and 58 were parsimony-informative. However, the ITS dataset contained $634 \mathrm{bp}$ with only 31 parsimony informative sites (Table 2). It is obvious that the plastome datasets comprised more variable sites than the ITS dataset but the variations rates were far lower than ITS datasets (Table 2).

Phylogenetic trees using ML and BI methods done on each dataset resulted in similar topologies and we obtained almost the same topological divergences for all samples based on the PCGs and non-coding region datasets (Fig. $4 \mathrm{~b}$ and $\mathrm{c}$ ). The phylogenetic tree derived from the whole plastomes dataset differed slightly from the other two plastome trees. In the former tree, phylogenetic relationships among five subclades (i.e. Ore1, Oja1, Oja2-5, Oja5-8 and Och4-5) were resolved, while relationships among these five subclades were not clear in 


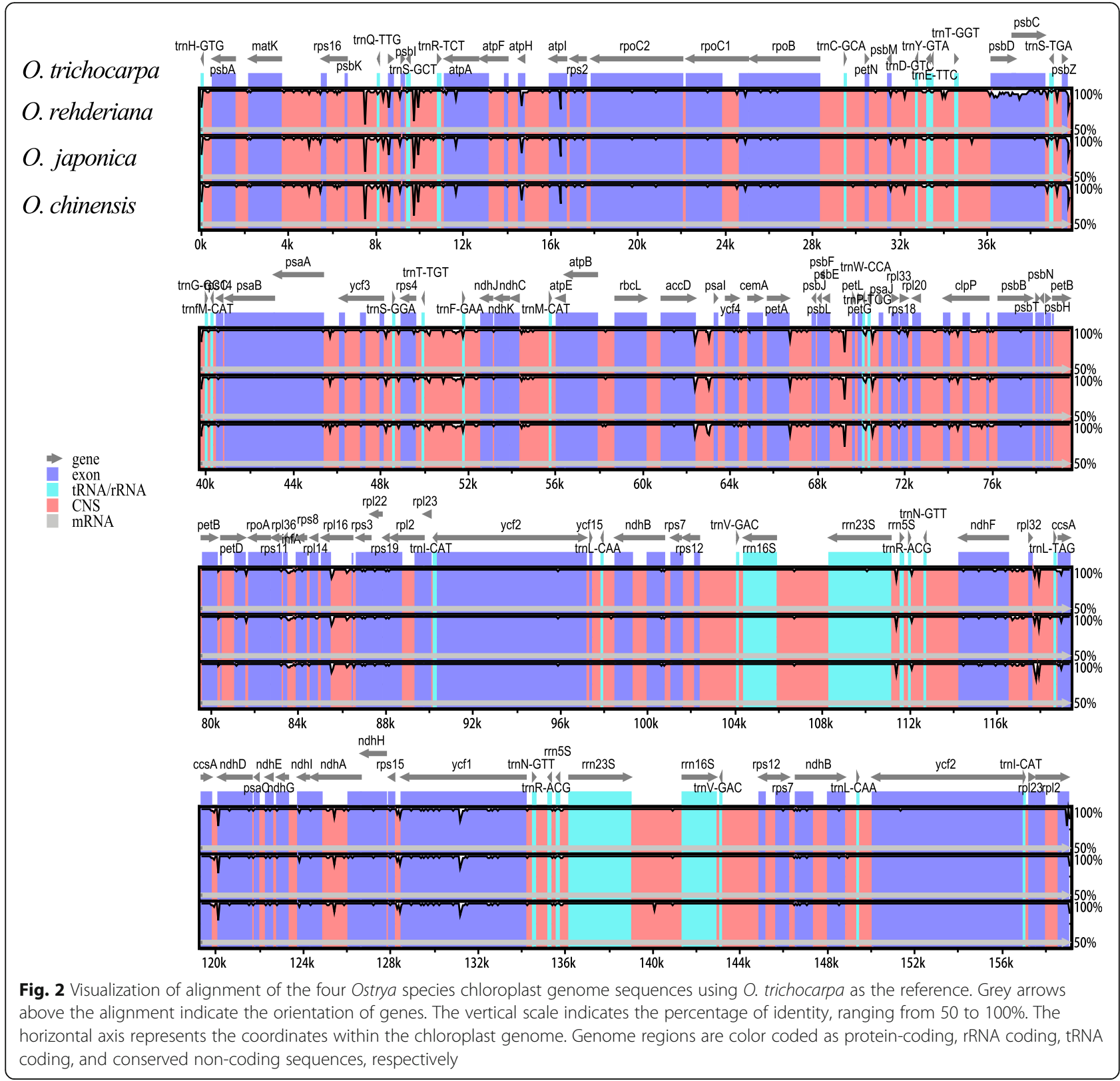

the later two trees (Fig. 4a, b and c). In general, except for the samples of $O$. rehderiana that collected from the same population, the other three species were all non-monophyletic in the plastome trees (Fig. $4 \mathrm{a}, \mathrm{b}$ and c). O. japonica was separated into four subclades, while the other two species (O. chinensis and O. trichocarpa) formed another two subclades, respectively. Moreover, some samples that were collected from adjacent regions regardless of species may be clustered together in the plastome trees. For example, population Otr1 of O. trichocarpa was geographically close to populations Och2 and Och3 of O. chinensis (Additional file 6: Figure S1), and three samples of Otr1 were grouped with samples from populations Och2 and Och3. The heatmap of plastome distances was consistent with such failure of delimitation of interspecific relationships (Additional file 7: Figure S2). However, the ITS tree contrasted greatly with that inferred from the plastomes and the four species were all well-delimitated with high support values in the ITS phylogenetic tree (Fig. 4d).

\section{Discussion}

In the present study, we determined whole plastid genomes of four Ostrya species, which provides significant genetic resources and facilitate to comprehend the plastid genome evolution. The exhibition of typical angiosperm quadripartite structure and highly conserved gene content, gene order and GC content in Ostrya plastomes 
Table 1 Characters of four Ostrya species chloroplast genomes

\begin{tabular}{|c|c|c|c|c|c|c|c|c|c|}
\hline ID & $\begin{array}{l}\text { Entire plastid } \\
\text { size (bp) }\end{array}$ & $\begin{array}{l}\text { Large single } \\
\text { copy (LSC) }\end{array}$ & $\begin{array}{l}\text { Small single } \\
\text { copy (SSC) }\end{array}$ & $\begin{array}{l}\text { Inverted } \\
\text { repeat (IR) }\end{array}$ & $\begin{array}{l}\text { GC content } \\
(\%)\end{array}$ & $\begin{array}{l}\text { Number of } \\
\text { genes }\end{array}$ & $\begin{array}{l}\text { Number of protein- } \\
\text { coding genes }\end{array}$ & $\begin{array}{l}\text { Number of } \\
\text { tRNA genes }\end{array}$ & $\begin{array}{l}\text { Number of } \\
\text { rRNA genes }\end{array}$ \\
\hline ore01 & 159,235 & 88,175 & 18,944 & 26,059 & 36.5 & 122 & 85 & 29 & 8 \\
\hline ore02 & 159,218 & 88,196 & 18,944 & 26,059 & 36.5 & 122 & 85 & 29 & 8 \\
\hline ore03 & 159,236 & 88,176 & 18,944 & 26,059 & 36.5 & 122 & 85 & 29 & 8 \\
\hline ore04 & 159,237 & 88,177 & 18,944 & 26,059 & 36.5 & 122 & 85 & 29 & 8 \\
\hline oja01 & 159,241 & 88,179 & 18,945 & 26,059 & 36.5 & 122 & 85 & 29 & 8 \\
\hline oja02 & 159,235 & 88,186 & 18,933 & 26,059 & 36.5 & 122 & 85 & 29 & 8 \\
\hline oja06 & 159,278 & 88,224 & 18,933 & 26,059 & 36.5 & 122 & 85 & 29 & 8 \\
\hline oja08 & 159,280 & 88,226 & 18,938 & 26,059 & 36.5 & 122 & 85 & 29 & 8 \\
\hline oja09 & 159,248 & 88,180 & 18,936 & 26,067 & 36.5 & 122 & 85 & 29 & 8 \\
\hline oja10 & 159,286 & 88,220 & 18,930 & 26,067 & 36.5 & 122 & 85 & 29 & 8 \\
\hline oja15 & 159,250 & 88,180 & 18,954 & 26,059 & 36.5 & 122 & 85 & 29 & 8 \\
\hline oja18 & 159,240 & 88,180 & 18,945 & 26,058 & 36.5 & 122 & 85 & 29 & 8 \\
\hline oja19 & 159,240 & 88,175 & 18,944 & 26,059 & 36.5 & 122 & 85 & 29 & 8 \\
\hline oja20 & 159,229 & 88,176 & 18,937 & 26,059 & 36.5 & 122 & 85 & 29 & 8 \\
\hline oja21 & 159,235 & 88,177 & 18,937 & 26,059 & 36.5 & 122 & 85 & 29 & 8 \\
\hline oja23 & 159,278 & 88,227 & 18,937 & 26,059 & 36.5 & 122 & 85 & 29 & 8 \\
\hline oja26 & 159,238 & 88,178 & 18,944 & 26,059 & 36.5 & 122 & 85 & 29 & 8 \\
\hline oja27 & 159,236 & 88,169 & 18,937 & 26,065 & 36.5 & 122 & 85 & 29 & 8 \\
\hline omu01 & 159,150 & 88,176 & 18,954 & 26,059 & 36.5 & 122 & 85 & 29 & 8 \\
\hline omu02 & 159,251 & 88,176 & 18,954 & 26,059 & 36.5 & 122 & 85 & 29 & 8 \\
\hline omu03 & 159,246 & 88,176 & 18,954 & 26,059 & 36.5 & 122 & 85 & 29 & 8 \\
\hline omu08 & 159,265 & 88,229 & 18,917 & 26,058 & 36.5 & 122 & 85 & 29 & 8 \\
\hline omu09 & 159,227 & 88,183 & 18,925 & 26,058 & 36.5 & 122 & 85 & 29 & 8 \\
\hline omu12 & 159,246 & 88,176 & 18,954 & 26,059 & 36.5 & 122 & 85 & 29 & 8 \\
\hline omu13 & 159,299 & 88,188 & 18,956 & 26,059 & 36.5 & 122 & 85 & 29 & 8 \\
\hline omu14 & 159,288 & 88,199 & 18,975 & 26,059 & 36.5 & 122 & 85 & 29 & 8 \\
\hline otr01 & 159,105 & 88,118 & 18,864 & 26,060 & 36.5 & 122 & 85 & 29 & 8 \\
\hline otr03 & 159,300 & 88,217 & 18,966 & 26,059 & 36.4 & 122 & 85 & 29 & 8 \\
\hline otr04 & 159,301 & 88,218 & 18,966 & 26,059 & 36.4 & 122 & 85 & 29 & 8 \\
\hline otr07 & 159,300 & 88,219 & 18,959 & 26,060 & 36.4 & 122 & 85 & 29 & 8 \\
\hline otr08 & 158,915 & 88,031 & 18,743 & 26,069 & 36.5 & 122 & 85 & 29 & 8 \\
\hline otr11 & 158,879 & 88,007 & 18,736 & 26,069 & 36.5 & 122 & 85 & 29 & 8 \\
\hline otr12 & 158,870 & 88,008 & 18,721 & 26,069 & 36.5 & 122 & 85 & 29 & 8 \\
\hline
\end{tabular}

were in accord with other members of Betulaceae [45-49]. The presence of repeats, especially in intergenic spacers, was often found associated with divergence regions [50, 51]. We found $57 \%$ of repeats occurred in intergenic spacers, which may play a role in maintaining interspecific divergence. In addition, the non-coding regions showed higher interspecific variations than the protein-coding regions as also found in many other groups, and phylogenetic analyses based on different regions may show inconsistence $[19,52]$, The conservatism of the Ostrya plastomes and well-aligned plastomes across different species can therefore facilitate the further phylogenetic analyses.

Previous studies based on a few cpDNA markers failed to resolve the interspecific relationships of Ostrya [53, 54], and surprisingly, our analyses based on three different plastome datasets (i.e. whole plastomes, PCGs and non-coding regions) with obviously more informative sites still failed to do so, which is contrast to most plastome studies that recovered high phylogenetic resolution and resolved relationships [55-58]. However, it should be 


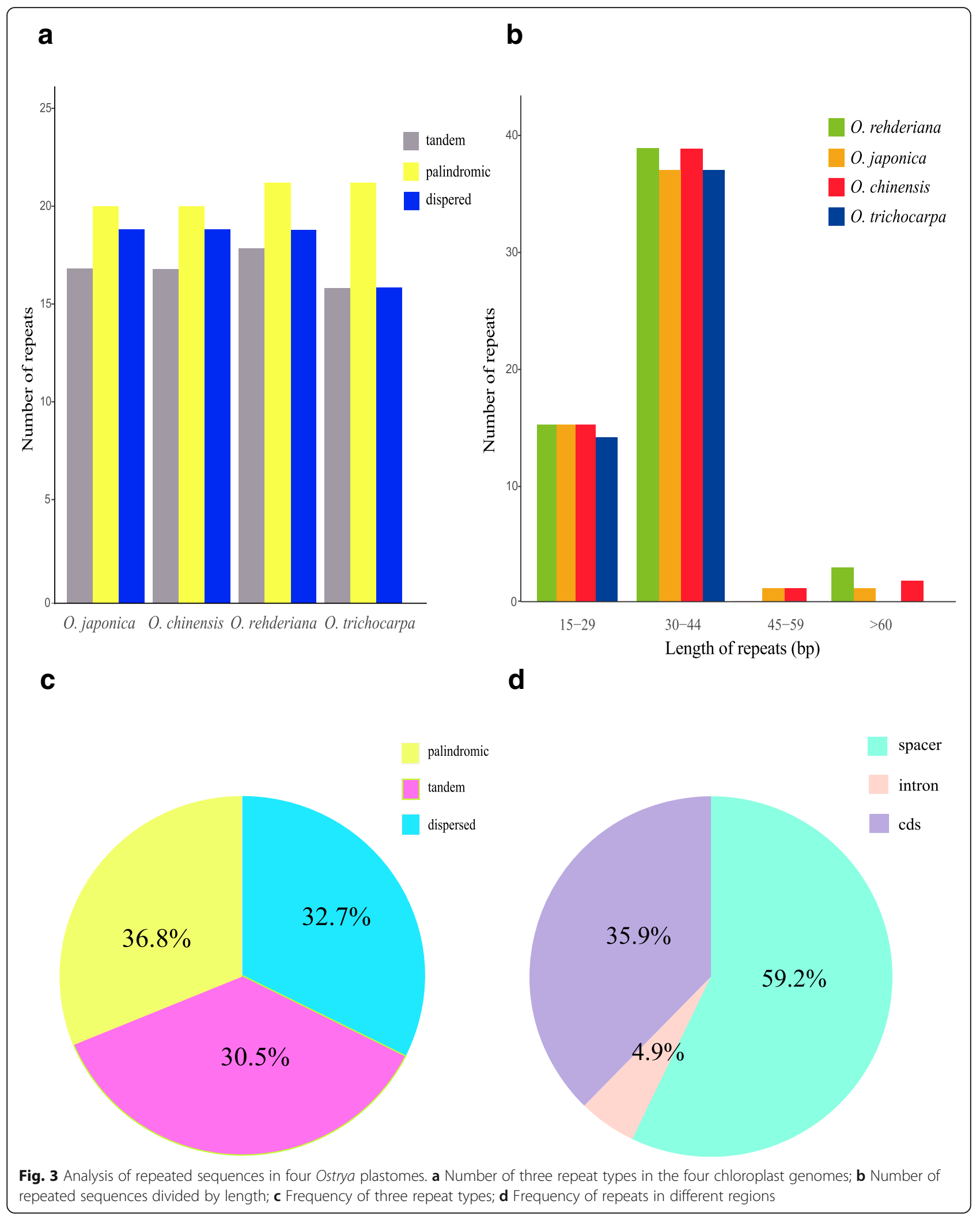


Table 2 Number of informative sites in different datasets

\begin{tabular}{lllll}
\hline Locus & Whole plastomes $(\mathrm{bp})$ & Protein-coding genes (aa) & Non-coding regions (bp) & ITS (bp) \\
\hline Constant sites & 154,907 & 21,492 & 63,147 & 570 \\
Parsimony informative sites & 456 & 58 & 244 & 31 \\
Variable sites & 2209 & 228 & 1332 & 64 \\
Total sites & 157,116 & 21,720 & 64,479 & 634 \\
Variation rates $/ \%$ & 1.41 & 1.05 & 2.07 & 10.09 \\
\hline
\end{tabular}

For whole plastomes, non-coding regions and ITS datasets, nucleotide sequences were used, while for protein-coding genes, amino acid sequences were used. $b p$ base pair, aa amino acid

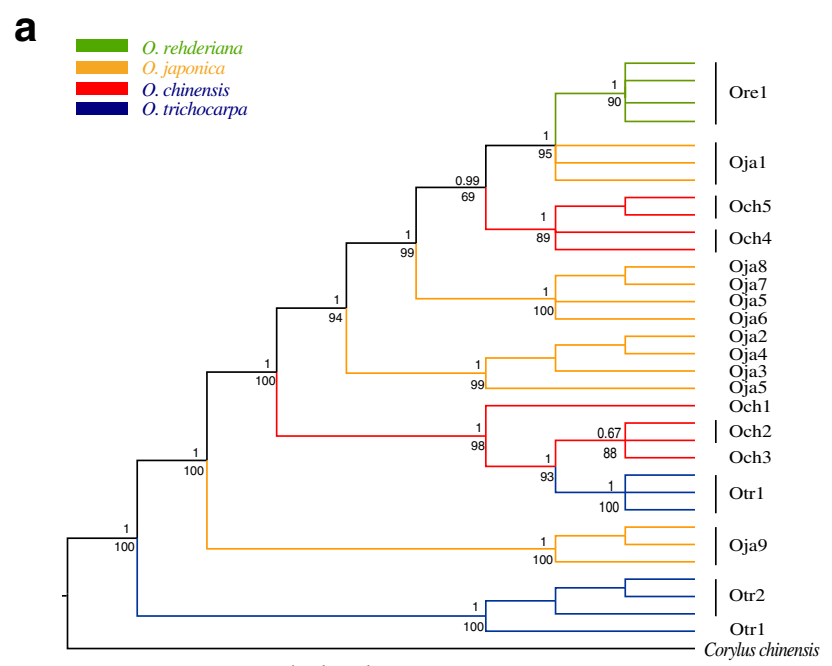

Whole plastome sequences

C

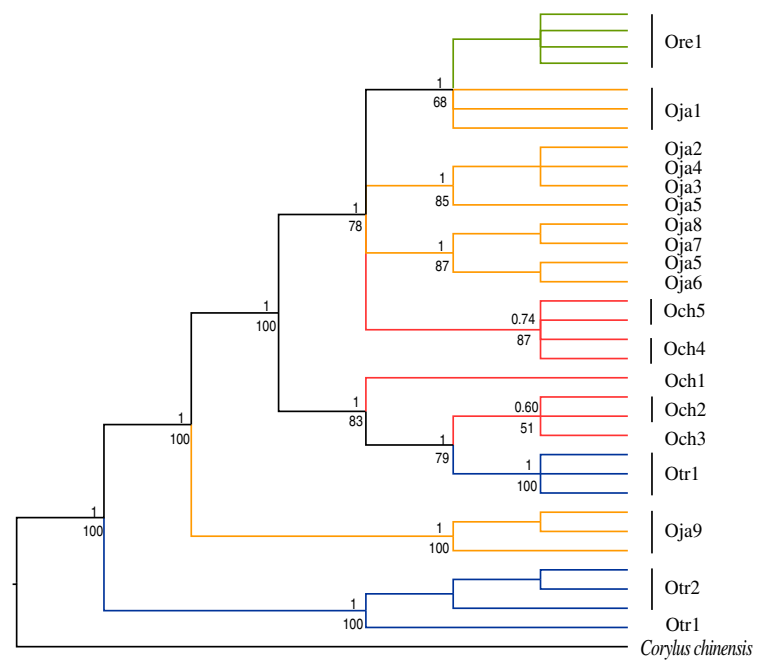

Non-coding regions b

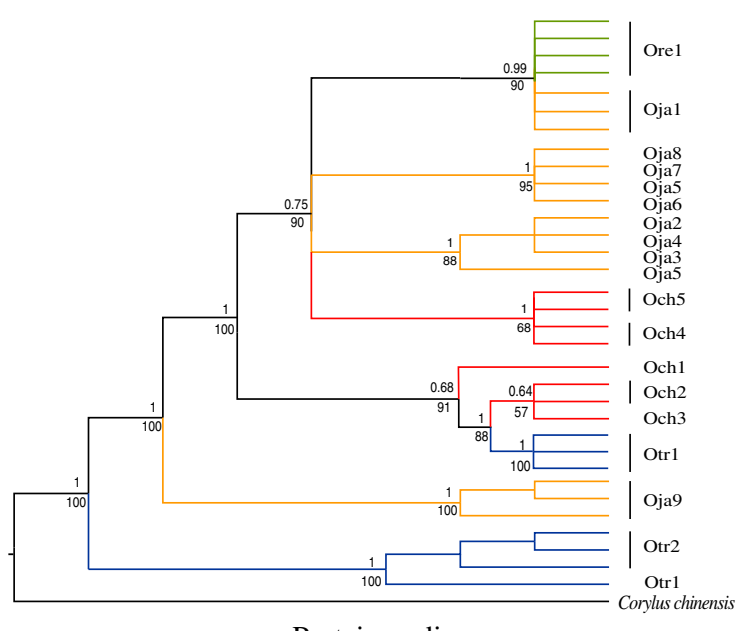

Protein-coding genes

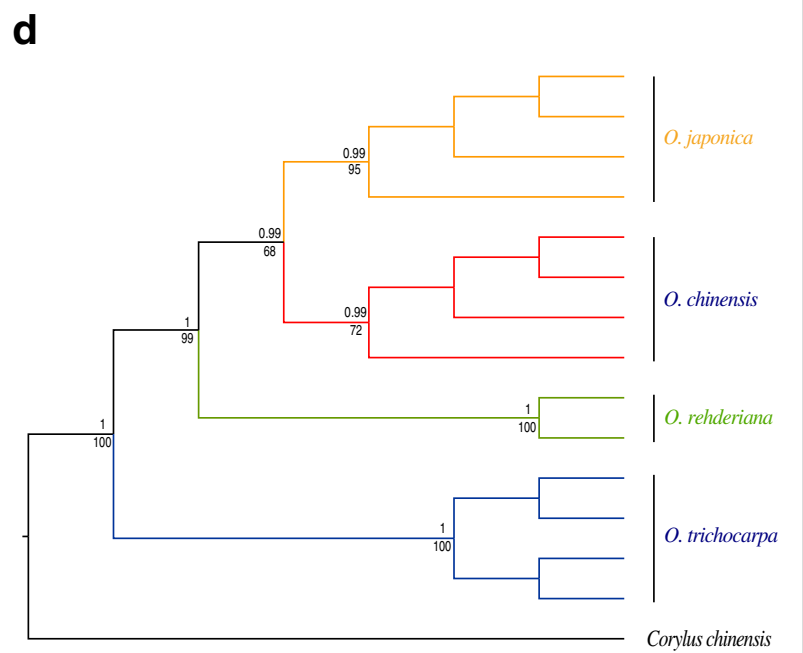

ITS sequences

Fig. 4 Cladogram of Ostrya species using Bayesian inference (BI) analysis and Maximum likelihood (ML) analysis based on different datasets. Support values are shown for nodes as BI posterior probability (above branches) /ML bootstrap (below branches). The four species are shown by different colors. a - c plastome trees based on different regions; population information for each species is shown at the tips. $\mathbf{d}$ nuclear ITS (Internal transcribed spacer) tree; samples for each species are selected from different populations 
noted that multiple individuals from different populations per species were included in our analyses, and these samples seem to be clustered by geography other than species (Fig. 4, Additional file 6: Figure S1). These results further indicate that phylogenetic studies based on plastome using only one individual per species are not appropriate, especially among closely related species. For example, using one individual from either population Oja9 or Oja1 of $O$. japonica will lead to very different interspecific relationships to other species (Fig. 4).

By contrast, ITS sequences with fewer informatic sites compared with the plastome datasets (Table 2) successfully discriminate the four species. The incongruence between nuclear ITS and cpDNA trees is common observed in many other plants $[9,59,60]$ and usually can be explained as follows: firstly, the nuclear ITS sequence has higher mutation rate than chloroplast genome [61] and is biparental inherited with both pollen and seeds dispersion, while chloroplast of most angiosperm is maternal inherited and dispersed only by seeds $[62,63]$. Chloroplast DNA with relatively low rates of intraspecific gene flow should be more introgressed, which lead to transfer of genetic material across species boundaries [64]. This may explain why some samples belonging to different species collected from adjacent regions are grouped together in the plastome trees (Fig. 4). In contrast, nuclear loci that experience high rates of intraspecific gene flow should enhance species delimitation [65]. We find the same pattern in our results that the ITS tree has much clearer species delimitation than the plastome trees (Fig. 4). However, alternative scenario may occur in other systems, for example, in Orychophragmus [66] and in Primula section Armerina [67], where cpDNA was highly effective in discriminating closely related species, but nrDNA failed. In addition, incomplete lineage sorting occurred during the fast-radiative speciation of this genus may also play a role in the cyto-nuclear discordance [60,68]. However, our data is not appropriate to clearly discriminate among these possible scenarios, we therefore recognize that further studies involving whole genome sequences at the population level are needed to better understand their evolutionary histories.

\section{Conclusions}

Our phylogenetic analyses based on plastome datasets still failed to resolve the interspecific delimitation and relationships among four closely related Ostrya species, in contrast to the well-resolved phylogeny based on the ITS sequence variations. Such incongruence may result from incomplete lineage sorting and hybrid introgression during the divergences of the four species. Previous plastome studies using one individual per species usually recovered high phylogenetic resolution and resolved relationships, however, our results indicated that multiple samples from different populations should be considered when doing such phylogenetic studies, especially among closely related species. Finally, our findings shed light on some interesting evolutionary questions, e.g., what causes O. japonica split into four subclades in the plastome trees, and further genomic studies at the population level are necessary to gain a deep understanding of the evolution of these species.

\section{Additional files}

Additional file 1: Table S1. Sample information of Ostrya populations. (DOCX $25 \mathrm{~kb}$ )

Additional file 2: Table S2. 33 complete chloroplast genomes of four Ostrya species from GenBank. (DOCX 17 kb)

Additional file 3: Table S3. Best-fit models for each of the amino acid sequence of PCGs. (DOCX $27 \mathrm{~kb}$ )

Additional file 4: Table S4. Gene list of plastomes of four Ostrya species. (DOCX $21 \mathrm{~kb}$ )

Additional file 5: : Table S5. Repeats distribution in Ostrya chloroplast genome. (XLSX $17 \mathrm{~kb}$ )

Additional file 6: Figure S1. Sample information of Ostrya populations. Different dot color indicates the different taxa. Green: O. rehderiana. Orange: O. japonica. Red: O. chinensis. Blue: O. trichocarpa. The permission of the graph is not required. (PDF $9547 \mathrm{~kb}$ )

Additional file 7: Figure S2. Heatmap of plastome distances among 17 Ostrya populations. For populations that have multiple samples, we only select one sample in this analysis. (PDF $375 \mathrm{~kb}$ )

\section{Abbreviations}

aa: Amino acid; BI: Bayesian inference; bp: Base pair; cpDNA: chloroplast DNA: GC: Guanine cytosine; IR: Inverted repeat region; ITS: Internal transcribed spacer; LSC: Large single copy region; ML: Maximum likelihood; PCGs: Protein coding genes; SSC: Small single copy region

\section{Acknowledgements}

We would like to thank Ying Li, Xu Zhang and Jianchao Ma for their technical help in analyzing data.

\section{Funding}

Financial support was provided by the National Natural Science Foundation of China (41471042), National Key Research and Development Program (2017YFC0505203), National Key Project for Basic Research (2012CB114504), Ministry of Science and Technology of the People's Republic of China (2010DFA34610), and International Collaboration 111 Projects of China. The funding body did not contribute to the design of the study or collection, analysis and interpretation of data and writing the manuscript.

\section{Availability of data and materials}

All plastome sequence data that support the findings of this study have been deposited in GenBank (accession numbers can be found in Additional file 2: Table S2). We also uploaded all our alignment and tree files to TreeBase, which can be found in http://purl.org/phylo/treebase/phylows/study/TB2:S23574.

\section{Authors' contributions}

YJ and GR designed this study. ZL, YY and DW collected the samples. YJ and ZL extracted total genomic DNA. YJ, YY and DW analyzed the data, YJ and $Y Y$ wrote the manuscript. DW and GR revised the manuscript. All of the authors read and agreed to the manuscript and this submission.

\section{Ethics approval and consent to participate}

We received ethics approval for the sampling of the populations of this plant from Tianmu Mountain National Nature Reserve and Maolan National Nature Reserve. During the sampling process, we strictly abided by the rules and 
regulations according to IUCN Policy Statement on Research Involving Species at Risk of Extinction, collected a small amount of leaves that did not cause damage to the plants, which was carried out under the supervision of the management personnel of the reserve.

\section{Consent for publication}

\section{Not applicable.}

\section{Competing interests}

The authors declare that they have no competing interests.

\section{Publisher's Note}

Springer Nature remains neutral with regard to jurisdictional claims in published maps and institutional affiliations.

Received: 16 January 2018 Accepted: 1 March 2019

Published online: 12 March 2019

\section{References}

1. Korkut DS, Korkut S. Determination of the shear and cleavage strengths of European Hophornbeam (Ostrya carpinifolia Scop.) wood. Beykent University J Sci Technol. 2008;2(1):131-7.

2. Alden HA. Hardwoods of North America, vol. 83. Madison: US Department of Agriculture, Forest Service, Forest Products Laboratory; 1995.

3. Doğu AD, Kartal SN, Köse C, Erdin N. Some anatomical properties and wood density of Ostrya carpinifolia Scop. J Faculty Forestry Istanbul Univ İstanbul Üniversitesi Orman Fakültesi Dergisi. 2000;50(2):167-76.

4. Korkut S, Guller B. Physical and mechanical properties of European Hophornbeam (Ostrya carpinifolia Scop.) wood. Bioresour Technol. 2008; 99(11):4780-5.

5. Lu Z, Zhang D, Liu S, Yang X, Liu X, Liu J. Species delimitation of Chinese hop-hornbeams based on molecular and morphological evidence. Ecol Evol. 2016:6(14):4731-40.

6. Turner IM. Names of extant angiosperm species that are illegitimate homonyms of fossils. In: Ann Bot Fenn: 2014. BioOne: 305-317.

7. Ren H, Zhang Q, Lu H, Liu H, Guo Q, Wang J, Jian S. Wild plant species with extremely small populations require conservation and reintroduction in China. Ambio. 2012;41(8):913-7.

8. Schluter D. The ecology of adaptive radiation. Oxford: OUP; 2000

9. Arnold ML. Evolution through genetic exchange. Oxford: Oxford University Press; 2006

10. Suo $Z$, Chen $L$, Pei $D$, Jin $X$, Zhang $H$. A new nuclear DNA marker from ubiquitin ligase gene region for genetic diversity detection of walnut germplasm resources. Biotechnol Rep. 2015:5:40-5.

11. Moore MJ, Soltis PS, Bell CD, Burleigh JG, Soltis DE. Phylogenetic analysis of 83 plastid genes further resolves the early diversification of eudicots. Proc Natl Acad Sci U S A. 2010;107(10):4623-8.

12. Parks $M$, Cronn $R$, Liston $A$. Increasing phylogenetic resolution at low taxonomic levels using massively parallel sequencing of chloroplast genomes. BMC Biol. 2009;7(1):84.

13. Ruhsam M, Rai HS, Mathews S, Ross TG, Graham SW, Raubeson LA, Mei W, Thomas PI, Gardner MF, Ennos RA. Does complete plastid genome sequencing improve species discrimination and phylogenetic resolution in Araucaria? Mol Ecol Resour. 2015;15(5):1067-78.

14. Yang J-B, Tang M, Li H-T, Zhang Z-R, Li D-Z. Complete chloroplast genome of the genus Cymbidium: lights into the species identification, phylogenetic implications and population genetic analyses. BMC Evol Biol. 2013;13(1):84.

15. Jansen RK, Cai Z, Raubeson LA, Daniell H, Leebens-Mack J, Müller KF, Guisinger-Bellian M, Haberle RC, Hansen AK, Chumley TW. Analysis of 81 genes from 64 plastid genomes resolves relationships in angiosperms and identifies genome-scale evolutionary patterns. Proc Natl Acad Sci U S A. 2007;104(49):19369-74.

16. Nock CJ, Waters DL, Edwards MA, Bowen SG, Rice N, Cordeiro GM, Henry RJ. Chloroplast genome sequences from total DNA for plant identification. Plant Biotechnol J. 2011;9(3):328-33

17. Moore MJ, Dhingra A, Soltis PS, Shaw R, Farmerie WG, Folta KM, Soltis DE. Rapid and accurate pyrosequencing of angiosperm plastid genomes. BMC Plant Biol. 2006:6(1):17

18. Tangphatsornruang S, Somta P, Uthaipaisanwong P, Chanprasert J, Sangsrakru D, Seehalak W, Sommanas W, Tragoonrung S, Srinives P. Characterization of microsatellites and gene contents from genome shotgun sequences of mungbean (Vigna radiata (L.) Wilczek). BMC Plant Biol. 2009;9(1):137.

19. Hu H, Hu Q, Al-Shehbaz IA, Luo X, Zeng T, Guo X, Liu J. Species delimitation and interspecific relationships of the genus Orychophragmus (Brassicaceae) inferred from whole chloroplast genomes. Front Plant Sci. 2016;7:1826.

20. Doyle J. A rapid DNA isolation procedure for small quantities of fresh leaf tissue. Phytochem Bull. 1987;19(1):11-5.

21. Bolger AM, Lohse M, Usadel B. Trimmomatic: a flexible trimmer for Illumina sequence data. Bioinformatics. 2014;30(15):2114-20.

22. Langmead B, Salzberg SL, Langmead B, Salzberg SL. Fast gapped-read alignment with bowtie 2. Nat Methods. 2012;9:357-9 9(4):357-359.

23. Li H, Handsaker B, Wysoker A, Fennell T, Ruan J, Homer N, Marth G, Abecasis G, Durbin R. The sequence alignment/map format and SAMtools. Bioinformatics. 2009;25(16):2078-9.

24. Zerbino DR, Birney E. Velvet: algorithms for de novo short read assembly using de Bruijn graphs. Genome Res. 2008;18(5):821-9.

25. Huang DI, Cronk QC. Plann: a command-line application for annotating plastome sequences. Appl Plant Sci. 2015;3(8):1500026

26. Kearse M, Moir R, Wilson A, Stones-Havas S, Cheung M, Sturrock S, Buxton S, Cooper A, Markowitz S, Duran C. Geneious basic: an integrated and extendable desktop software platform for the organization and analysis of sequence data. Bioinformatics. 2012;28(12):1647-9.

27. Lohse M, Drechsel O, Kahlau S, Bock R. OrganellarGenomeDRAW—a suite of tools for generating physical maps of plastid and mitochondrial genomes and visualizing expression data sets. Nucleic Acids Res. 2013;41(W1):W575-81.

28. Frazer KA, Pachter L, Poliakov A, Rubin EM, Dubchak I. VISTA: computational tools for comparative genomics. Nucleic Acids Res. 2004;32(suppl 2):W273-9.

29. Kurtz S, Choudhuri JV, Ohlebusch E, Schleiermacher C, Stoye J, Giegerich R. REPuter: the manifold applications of repeat analysis on a genomic scale. Nucleic Acids Res, 2001:29(22):4633-42.

30. Kurtz S, Schleiermacher C. REPuter: fast computation of maximal repeats in complete genomes. Bioinformatics. 1999:15(5):426-7.

31. Benson G. Tandem repeats finder. A program to analyze DNA sequences. Nucleic Acids Res. 1999:27(2):573.

32. Grimm GW, Renner SS. Harvesting Betulaceae sequences from GenBank to generate a new chronogram for the family. Bot J Linn Soc. 2013;172(4):465-77.

33. Katoh K, Standley DM. MAFFT multiple sequence alignment software version 7: improvements in performance and usability. Mol Biol Evol. 2013;30(4):772-80.

34. Capella-Gutiérrez S, Silla-Martínez JM, Gabaldón T. trimAl: a tool for automated alignment trimming in large-scale phylogenetic analyses. Bioinformatics. 2009;25(15):1972-3.

35. Di Tommaso P, Moretti S, Xenarios I, Orobitg M, Montanyola A, Chang J-M, Taly J-F, Notredame C. T-coffee: a web server for the multiple sequence alignment of protein and RNA sequences using structural information and homology extension. Nucleic Acids Res. 2011;39:W13-7.

36. Kück P, Longo GC. FASconCAT-G: extensive functions for multiple sequence alignment preparations concerning phylogenetic studies. Front Zool. 2014;11(1):81.

37. Posada D. jModelTest: phylogenetic model averaging. Mol Biol Evol. 2008; 25(7):1253-6.

38. Stamatakis A. RAxML version 8: a tool for phylogenetic analysis and postanalysis of large phylogenies. Bioinformatics. 2014;30(9):1312-3.

39. Ronquist F, Teslenko M, Van Der Mark P, Ayres DL, Darling A, Höhna S, Larget B, Liu L, Suchard MA, Huelsenbeck JP. MrBayes 3.2: efficient Bayesian phylogenetic inference and model choice across a large model space. Syst Biol. 2012:61(3):539-42.

40. Darriba D, Taboada GL, Doallo R, Posada D. ProtTest 3: fast selection of bestfit models of protein evolution. Bioinformatics. 2011;27(8):1164-5.

41. Rambaut A, Suchard M, Xie D, Drummond A. Tracer v1. 6 http://beast. bio. ed. ac. uk. Tracer>(Online 2015, May 29) 2014

42. Meier-Kolthoff JP, Auch AF, Klenk H-P, Göker M. Genome sequence-based species delimitation with confidence intervals and improved distance functions. BMC Bioinformatics. 2013;14(1):60.

43. Cavalier-Smith T. Chloroplast evolution: secondary symbiogenesis and multiple losses. Curr Biol. 2002;12(2):R62-4.

44. Nie X, Lv S, Zhang Y, Du X, Wang L, Biradar SS, Tan X, Wan F, Weining S. Complete chloroplast genome sequence of a major invasive species, Crofton weed (Ageratina adenophora). PLoS One. 2012;7(5):e36869.

45. Yang Y, Wang M, Lu Z, Xie X, Feng S. Characterization of the complete chloroplast genome of Carpinus tientaiensis. Conserv Genet Resour. 2017;9(2):339-41.

46. Hu G, Cheng L, Lan Y, Cao Q, Huang W. The complete chloroplast genome sequence of Corylus chinensis Franch. Conserv Genet Resour. 2017;9(1):119-21. 
47. Hu G, Cheng L, Lan Y, Cao Q, Wang X, Huang W. The complete chloroplast genome sequence of the endangered Chinese endemic tree Corylus fargesii. Conserv Genet Resour. 2017;9(2):225-7.

48. Jansen RK, Ruhlman TA. Plastid genomes of seed plants. In: Genomics of chloroplasts and mitochondria. Netherlands: Springer; 2012. p. 103-26.

49. Choi KS, Park S. The complete chloroplast genome sequence of Aster spathulifolius (Asteraceae); genomic features and relationship with Asteraceae. Gene. 2015;572(2):214-21.

50. Wu F-H, Chan M-T, Liao D-C, Hsu C-T, Lee Y-W, Daniell H, Duvall MR, Lin C-S. Complete chloroplast genome of Oncidium Gower Ramsey and evaluation of molecular markers for identification and breeding in Oncidiinae. BMC Plant Biol. 2010;10(1):68.

51. Maier RM, Neckermann K, Igloi GL, Kössel H. Complete sequence of the maize chloroplast genome: gene content, hotspots of divergence and fine tuning of genetic information by transcript editing. J Mol Biol. 1995;251(5): 614-28.

52. Zhang Y-J, Ma P-F, Li D-Z. High-throughput sequencing of six bamboo chloroplast genomes: phylogenetic implications for temperate woody bamboos (Poaceae: Bambusoideae). PLoS One. 2011;6(5):e20596.

53. Yoo KO, Wen J. Phylogeny of Carpinus and subfamily Coryloideae (Betulaceae) based on chloroplast and nuclear ribosomal sequence data. Plant Syst Evol. 2007:267(1-4):25-35

54. Yoo KO, Wen J. Phylogeny and biogeography of Carpinus and subfamily Coryloideae (Betulaceae). Int J Plant Sci. 2002;163(4):641-50

55. Huang Y, Li X, Yang Z, Yang C, Yang J, Ji Y. Analysis of complete chloroplast genome sequences improves phylogenetic resolution in Paris (Melanthiaceae). Front Plant Sci. 2016;7:1797.

56. Zeng S, Zhou T, Han K, Yang Y, Zhao J, Liu Z-L. The complete chloroplast genome sequences of six Rehmannia species. Genes. 2017:8(3):103.

57. Liu L-X, Li R, Worth JR, Li X, Li P, Cameron KM, Fu C-X. The complete chloroplast genome of Chinese bayberry (Morella rubra, Myricaceae) implications for understanding the evolution of Fagales. Front Plant Sci. 2017;8:968.

58. Li Y, Yang Y, Yu L, Du X, Ren G. Plastomes of nine hornbeams and phylogenetic implications. Ecol Evol. 2018;8:8770-8.

59. Morando M, Avila LJ, Baker J, Sites JW Jr. Phylogeny and phylogeography of the Liolaemus darwinii complex (Squamata: Liolaemidae): evidence for introgression and incomplete lineage sorting. Evolution. 2004;58(4):842-61.

60. Pollard DA, lyer VN, Moses AM, Eisen MB. Widespread discordance of gene trees with species tree in Drosophila: evidence for incomplete lineage sorting. PLoS Genet. 2006;2(10):e173.

61. Yang J, Yue M, Niu C, Ma X-F, Li Z-H. Comparative analysis of the complete chloroplast genome of four endangered herbals of Notopterygium. Genes. 2017;8(4):124

62. Hagemann R. The sexual inheritance of plant organelles. Molecular biology and biotechnology of plant organelles; 2004. p. 93-113.

63. Reboud X, Zeyl C. Organelle inheritance in plants. Heredity. 1994;72(2):132-40.

64. Du FK, Petit RJ, Liu JQ. More introgression with less gene flow: chloroplast vs. mitochondrial DNA in the Picea asperata complex in China, and comparison with other conifers. Mol Ecol. 2009;18(7):1396-407.

65. Petit RJ, Excoffier L. Gene flow and species delimitation. Trends Ecol Evol. 2009;24(7):386-93.

66. Hu H, Al-Shehbaz IA, Sun Y, Hao G, Wang Q, Liu J. Species delimitation in Orychophragmus (Brassicaceae) based on chloroplast and nuclear DNA barcodes. Taxon. 2015;64(4):714-26.

67. Ren G, Conti E, Salamin N. Phylogeny and biogeography of Primula sect. Armerina: implications for plant evolution under climate change and the uplift of the Qinghai-Tibet plateau. BMC Evol Biol. 2015;15(1):161.

68. Carstens BC, Knowles LL. Estimating species phylogeny from gene-tree probabilities despite incomplete lineage sorting: an example from Melanoplus grasshoppers. Syst Biol. 2007;56(3):400-11.

\section{Ready to submit your research? Choose BMC and benefit from:}

- fast, convenient online submission

- thorough peer review by experienced researchers in your field

- rapid publication on acceptance

- support for research data, including large and complex data types

- gold Open Access which fosters wider collaboration and increased citations

- maximum visibility for your research: over $100 \mathrm{M}$ website views per year

At BMC, research is always in progress.

Learn more biomedcentral.com/submissions 